\title{
CAFE: a new, improved nonresonant laser-induced fluorescence instru- ment for airborne in situ measurement of formaldehyde
}

Jason M. St. Clair et al.

Correspondence to: Jason M. St. Clair (jason.m.stclair@nasa.gov)

The copyright of individual parts of the supplement might differ from the CC BY 4.0 License. 


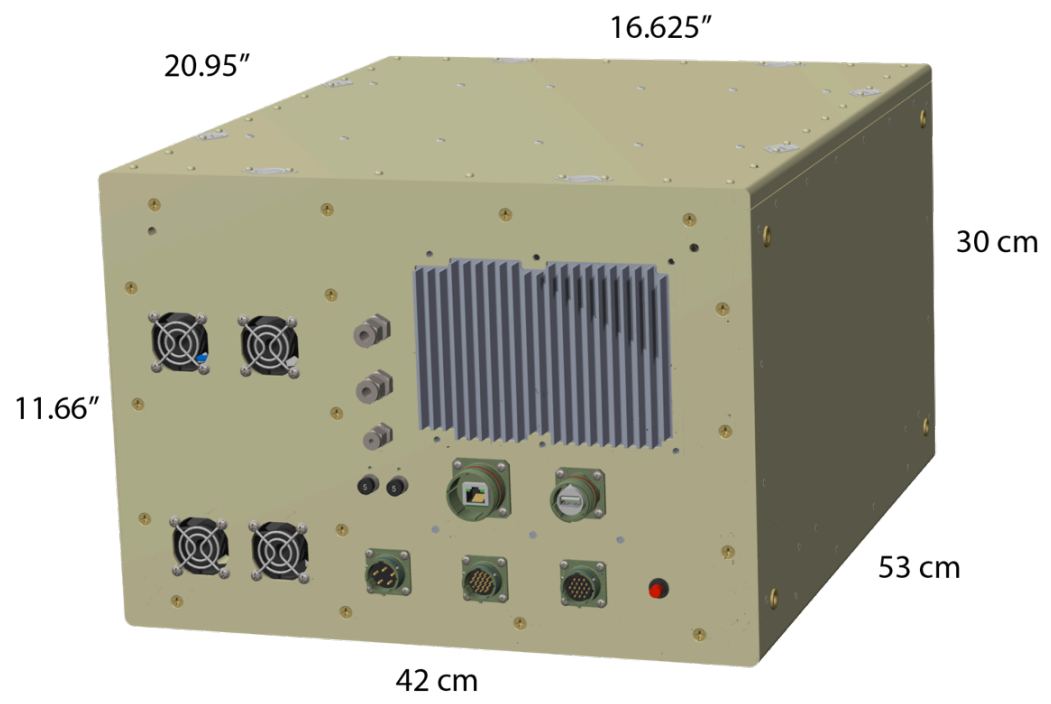

Figure S1. CAFE instrument chassis and dimensions.

\section{Measurement interference from $\mathrm{H}_{2} \mathrm{O}$ Raman}

During a limited number of AJAX flights with the COFFEE instrument, the standard data processing routine resulted in negative $\mathrm{HCHO}$ data for parts of the flight. The negative data was the consequence of high signal coincident with the laser pulse that decayed more rapidly in time than the air exemplar. The two-parameter fit overestimated the air exemplar scalar in order to balance the high signal early in the fit window, resulting in a scaled air exemplar higher than the data at the end of the fit window which was offset by a negative scalar for the HCHO exemplar. The short-term solution to the obviously incorrect negative data was to shift the start of the fitting window to a later time, reducing the effect of the measurement interference but also reducing the measurement precision. The shifted fitting window became the standard approach for processing COFFEE data. When it became clear that the negative $\mathrm{HCHO}$ data occurred during periods of high ambient water vapor, the possibility of $\mathrm{H}_{2} \mathrm{O}$ Raman causing the interference was investigated in the laboratory. UHP air was passed through a water bubbler to provide humidity to CAFE and the cause of the interference was confirmed. New long-pass optical filters were selected to block the water Raman signal and installed on CAFE and COFFEE. Fig. S2 shows wavelength scans of the PMT signal with three optical filter combinations that demonstrate the suppression of the $\mathrm{H}_{2} \mathrm{O}$ Raman signal with the new filters. The Raman features are, left to right, $\mathrm{N}_{2}, \mathrm{O}_{2}$ and $\mathrm{H}_{2} \mathrm{O}$. The cell pressure for these scans was 600 Torr and the scans were preformed using an Oriel monochromator (model 78025). 


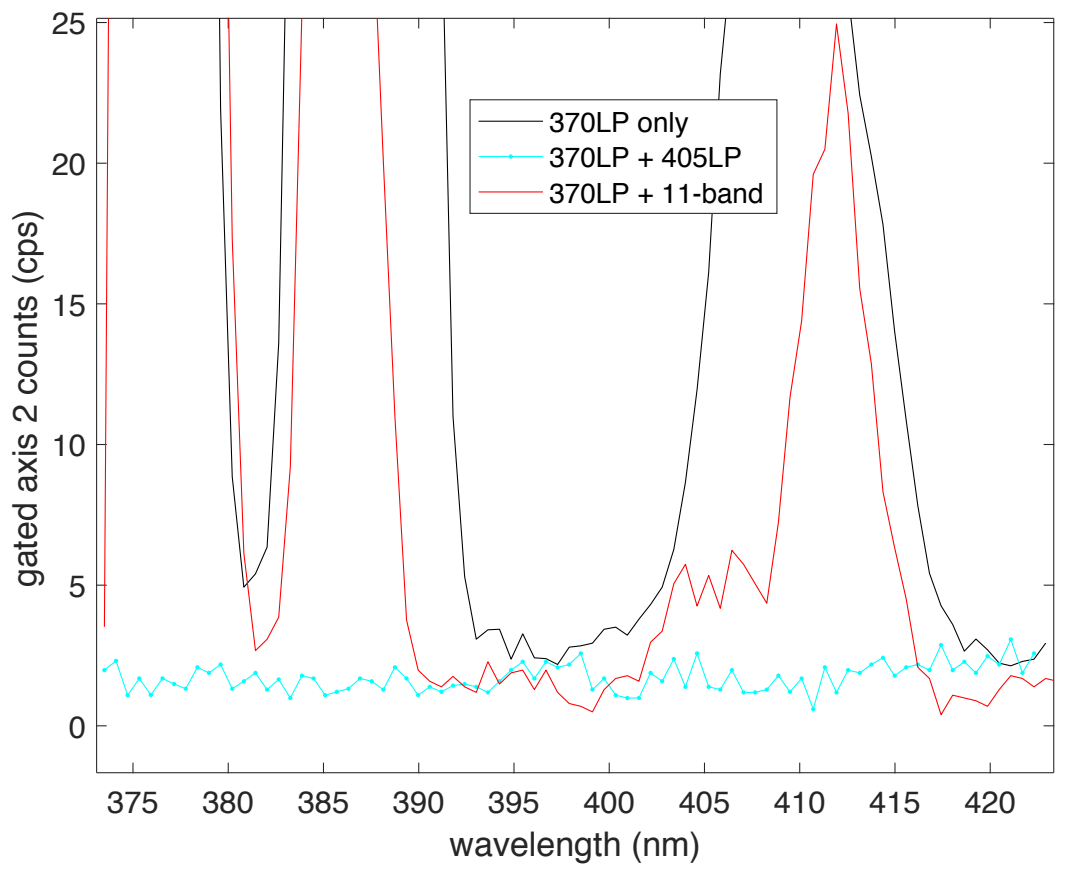

Figure S2. The $405 \mathrm{LP}$ is very effective at blocking the $\mathrm{H}_{2} \mathrm{O}$ Raman signal at $\sim 410 \mathrm{~nm}$. 

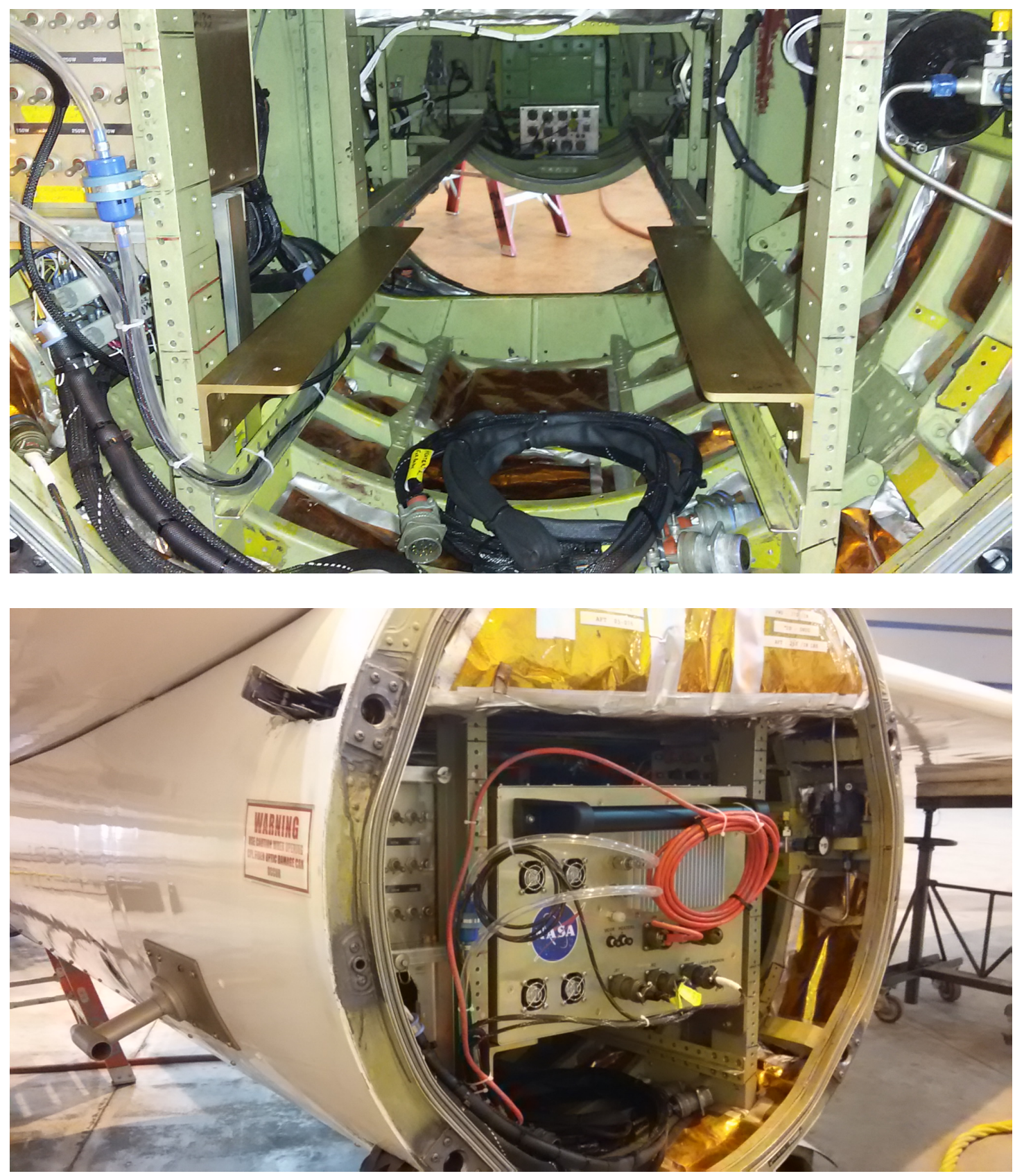

Figure S3. CAFE installation on the NASA ER-2: (top) mounting angles installed in the midbody of the left superpod and (bottom) CAFE fully installed for engineering test flights in 2016. The CAFE inlet is visible in the lower left of the bottom photo. 